\title{
Serum magnesium level is not associated with inflammation in patients with knee osteoarthritis
}

\author{
İlke Coşkun Benlidayı, ${ }^{1}$ Neslihan Gökçen, ${ }^{2}$ Tunay Sarpel ${ }^{1}$ \\ ${ }^{1}$ Department of Physical Medicine and Rehabilitation, Cukurova University Faculty of Medicine, Adana, Turkey \\ ${ }^{2}$ Department of Physical Medicine and Rehabilitation, Division of Rheumatology, Cukurova University Faculty of Medicine, Adana, Turkey \\ Received: May 2016 Accepted: July 2016
}

\begin{abstract}
Objectives: In this study, we aimed to evaluate the relationship of serum magnesium $(\mathrm{Mg})$ with the radiological severity and inflammation in the patients with knee osteoarthritis (OA).

Patients and methods: A total of 75 patients (61 females, 14 males; mean age $67.5 \pm 9.9$ years; range 44 to 92 years) with the Kellgren-Lawrence Grade 1-4 knee OA were included in the study. Serum Mg levels $(\mathrm{mg} / \mathrm{dL})$ and inflammatory markers including erythrocyte sedimentation rate $($ ESR; $\mathrm{mm} / \mathrm{h}$ ) and C-reactive protein (CRP; $\mathrm{mg} / \mathrm{dL})$ were recorded. Serum Mg levels of the patients with mild OA (Grade 1-2) and severe OA (Grade 3-4) were compared. The correlation between $\mathrm{Mg}$ and both inflammatory markers was analyzed.

Results: Severe OA group had significantly lower Mg levels than the mild OA group $(\mathrm{p}=0.044)$. Serum Mg level was not found to be correlated with either inflammatory biomarkers.

Conclusion: Although serum Mg is associated with the radiological severity of knee OA, it is not associated with inflammatory biomarkers including ESR and CRP.
\end{abstract}

Keywords: C-reactive protein; erythrocyte sedimentation rate; inflammation; knee osteoarthritis; magnesium; osteoarthritis.

Magnesium $(\mathrm{Mg})$ is a critical micronutrient for the cellular function of the humans. It is dominantly located within the cartilage and bone of a human body. Magnesium not only takes place in protein and nucleic acid synthesis, but also is important for phosphorylation events. Therefore, in case of its deficiency, many physiological functions of the human cell may be impaired. ${ }^{[1]}$

Several studies have demonstrated that dietary $\mathrm{Mg}$ deficiency is linked with a number of health conditions including diabetes mellitus, hypertension, coronary heart disease, low bone density, and malignancies (colon and breast). ${ }^{[2-7]}$ However, there is a limited number of data whether $\mathrm{Mg}$ is associated with osteoarthritis (OA). ${ }^{[8-11]}$ Previous studies have primarily addressed into the association of $\mathrm{Mg}$ intake and radiographic knee OA. ${ }^{[8,10,11]}$ However, the direct relationship between serum $\mathrm{Mg}$ level and OA has been less studied. ${ }^{[9]}$ Aforementioned studies have shown that
$\mathrm{Mg}$ intake has an inverse relation with radiological knee OA. ${ }^{[8,10,11]}$ In a study, Zeng et al. ${ }^{[9]}$ suggested that there might be also an inverse relation with serum $\mathrm{Mg}$ concentrations and knee OA.

The association of $\mathrm{Mg}$ and knee $\mathrm{OA}$ has been attributed to the possible role of $\mathrm{Mg}$ on inflammation by the researchers. However, none has studied the direct relation of inflammatory markers with serum $\mathrm{Mg}$ concentration in knee OA. Therefore, in the present study, we aimed to investigate the relationship between serum $\mathrm{Mg}$ levels and both radiological severity and inflammatory markers in patients with knee OA.

\section{PATIENTS AND METHODS}

Ethical approval of the study was obtained from the Local Ethics Committee of Cukurova University. The study was conducted in accordance with the principles of the Declaration of Helsinki. 
In this retrospective study, knee OA patients who underwent anteroposterior knee radiography and biochemical evaluation of serum $\mathrm{Mg}$ level with the inflammatory biomarkers including erythrocyte sedimentation rate (ESR) and C-reactive protein (CRP) between January 2010 and July 2015 were assessed for eligibility. Inclusion criteria were as follows: an established diagnosis of at least Grade 1 knee OA according to the Kellgren-Lawrence (KL) radiographic grading system for $\mathrm{OA}^{[12]}$ and age above 40 years. Exclusion criteria were as follows: history of trauma and/or surgery to the lower extremities, any congenital and/or acquired skeletal deformities, malignancy, inflammatory rheumatic diseases (i.e., rheumatoid arthritis, ankylosing spondylitis, gout, and psoriatic arthritis), the presence of any reasons which might alter serum Mg levels (i.e., Mg supplementation, acute/chronic renal failure, and malabsorption syndromes), and inappropriate image quality of the knee radiographs.

Anteroposterior knee radiographs of the patients included in the study were evaluated on a computer screen using the Enlil PACS System-2.5 (Enlil PACS Viewer, Eroglu Yazılım, Eskisehir, Turkey). The radiological grade of knee OA was determined according to the $\mathrm{KL}$ radiographic grading system for $\mathrm{OA}^{\left[{ }^{[12]}\right.}$ The knees were, then, divided into two groups of knee OA. Knees with KL Grade 1-2 OA were accepted as mild OA, whereas those with KL Grade 3-4 OA were accepted as severe OA. All knees were graded once again by the first investigator to check the intraobserver reliability. The grading was also performed by a second investigator who was blind to the initial evaluation results to test the agreement between two observers.

Serum $\mathrm{Mg}$ level $(\mathrm{mg} / \mathrm{dL})$ with the inflammatory biomarkers including ESR $(\mathrm{mm} / \mathrm{h})$ and CRP $(\mathrm{mg} / \mathrm{dL})$ were recorded.

\section{Statistical analysis}

The statistical analysis was performed using the PASW for Windows 17.0 version software (SPSS Inc., Chicago, IL, USA). The distribution of the data was checked by the normality tests. Descriptive statistics were expressed in mean \pm standard deviation (SD) and median (min-max). The difference in $\mathrm{Mg}$ levels between two knee OA groups was analyzed by the independent samples t-test. The correlation of $\mathrm{Mg}$ with the inflammatory biomarkers was analyzed using the Spearman's correlation analysis. The Kappa ( $)$ statistics were used to test the intraobserver and interobserver reliabilities regarding the KL grading of knee OA. A $p$ value of $<0.05$ was considered statistically significant.

\section{RESULTS}

A total of 103 patients with knee OA were assessed for eligibility. Among them, 75 patients (61 females, 14 males) who remained after the application of the exclusion criteria were included in the study (Figure 1).

The mean age of the study population was $67.5 \pm 9.9$ (range 44 to 92$)$ years. Of the patients, $32.0 \%(n=24)$, $26.7 \%(\mathrm{n}=20), 18.7 \%(\mathrm{n}=14)$, and $22.7 \%(\mathrm{n}=17)$ had $\mathrm{KL}$ Grade 1, 2, 3, and 4 knee OA, respectively. Accordingly, $60 \%(n=45)$ and $40 \%(n=30)$ were assigned to the mild OA (KL Grade 1-2) and severe OA (KL Grade 3-4) groups, respectively. The interobserver reliability analysis showed a substantial agreement between two observers with a $\kappa$ value of $0.713 \pm 0.1(p<0.001)$. On the other hand, the intraobserver reliability was almost excellent $(\kappa=0.839 \pm 0.1, \mathrm{p}<0.001)$.

According to serum $\mathrm{Mg}$ levels between two groups, severe OA group had significantly lower Mg levels than the mild OA group with a mean serum Mg level of $1.96 \pm 0.1$ vs $2.03 \pm 0.2 \mathrm{mg} / \mathrm{dL}$, respectively $(\mathrm{p}=0.044)$ (Figure 2). However, there was no significant difference in none of the inflammatory biomarkers between the groups: the median (min-max) ESR values were 16 (range: 5 to 68 ) $\mathrm{mm} / \mathrm{h}$ and 21.5 (range: 2 to 47 ) $\mathrm{mm} / \mathrm{h}$ in mild and severe OA groups, respectively $(\mathrm{p}=0.144)$, whereas the median CRP values were 0.419 (range: 0.100 to 1.75 ) $\mathrm{mg} / \mathrm{dL}$ and 0.431 (range: 0.106 to 2.32 ) $\mathrm{mg} / \mathrm{dL}$ in mild and severe OA groups, respectively $(\mathrm{p}=0.741)$.

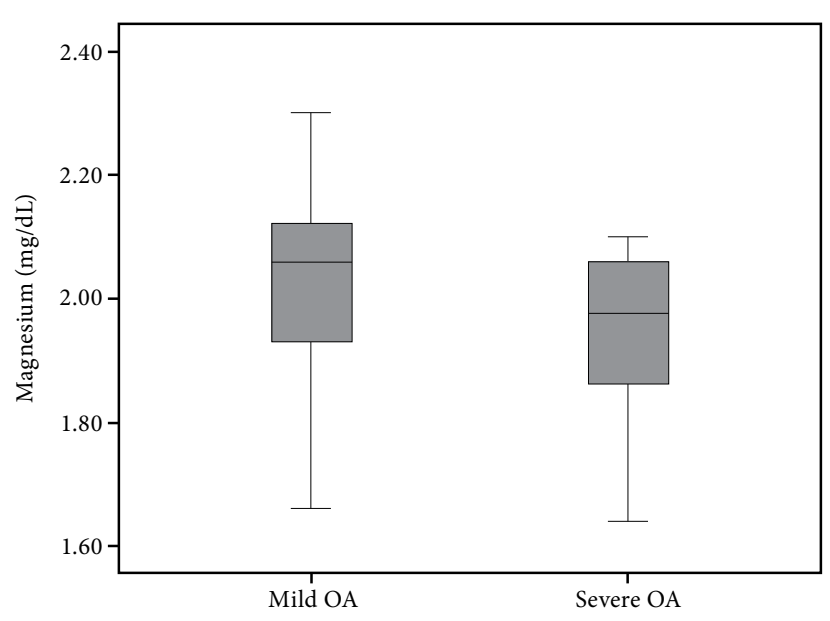

Figure 1. Comparison of serum magnesium level between mild and severe OA groups. OA: Osteoarthritis. 


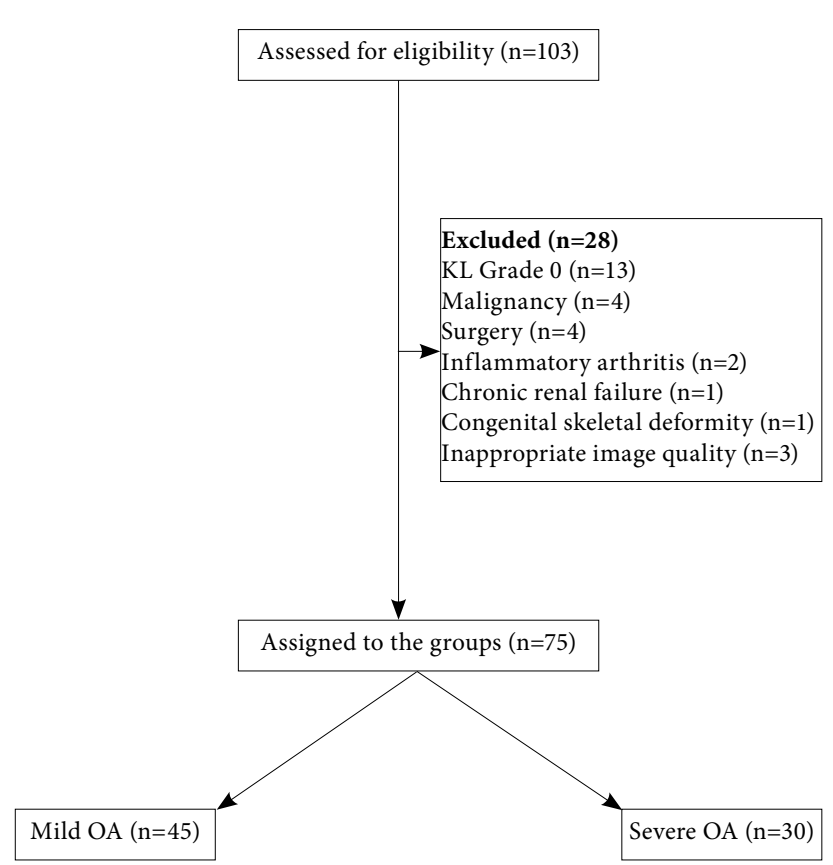

Figure 2. Flowchart of the study. KL: Kellgren-Lawrence; OA: Osteoarthritis.

In addition, serum $\mathrm{Mg}$ levels were not found to be correlated with inflammatory biomarkers including ESR and CRP $(\mathrm{r}=0.083[\mathrm{p}=0.480]$ and $\mathrm{r}=-0.007$ [p=0.952], respectively).

\section{DISCUSSION}

The present study revealed that, among patients with knee OA, those with severe disease pattern had lower Mg levels than those with mild knee OA and serum $\mathrm{Mg}$ levels were not associated with the inflammatory biomarkers.

In the literature, there is a limited number of studies investigating the effect of serum $\mathrm{Mg}$ on knee $\mathrm{OA}^{[9,11]} \mathrm{In}$ a cross-sectional study, Zeng et al..$^{[9]}$ found a significant inverse correlation between radiographic knee OA and serum Mg levels. Similarly, Hunter et al. ${ }^{[1]}$ showed lower $\mathrm{Mg}$ levels in female OA twins. In addition, the effect of dietary $\mathrm{Mg}$ on knee OA was examined by a number of researchers. ${ }^{[8,10]}$ Zeng et al ${ }^{[8]}$ demonstrated that higher $\mathrm{Mg}$ intake was associated with a lower risk of developing radiographic knee OA. The study by Qin et al. ${ }^{[10]}$ also showed a negative relationship between dietary Mg intake and radiographic knee OA in Caucasians.

The authors of the aforementioned studies proposed several explanations for the inverse relationship between serum $\mathrm{Mg}$ levels and knee OA.
They explained this relationship either through inflammation/immune mechanisms or the alterations in chondrocyte metabolism. However, the level of inflammatory biomarkers was not evaluated by the authors. Since a direct correlation between serum $\mathrm{Mg}$ levels and inflammatory biomarkers was not analyzed, these findings do not directly suggest that low serum $\mathrm{Mg}$ level enhances the risk of radiographic knee OA through its impact on inflammation. In the present study, however, we performed a correlation analysis between the inflammatory markers and serum Mg levels in knee OA patients, which showed no correlation with Mg levels and any of the measured markers. This finding indicates the potential catabolic role of decreased $\mathrm{Mg}$ level on the cartilage metabolism, thus, on the radiological progression in knee OA.

Regular provision of $\mathrm{Mg}$, which is an essential element for chondrocyte metabolism, is a must for the articular cartilage..$^{[1,13]}$ It is also necessary for the various chondrocyte nutrition states, including cell proliferation, re-differentiation, and chondrogenesis. ${ }^{[14]}$ In other words, $\mathrm{Mg}$ serves as a chondroprotective element by increasing the cell proliferation, protein expression, and growth factor efficacy. ${ }^{[13,14]}$ Therefore, the lack/depletion of $\mathrm{Mg}$ during cartilage development results in cartilage lesions. ${ }^{[15]}$ When the afore-mentioned evidences on $\mathrm{Mg}$ are synthesized with the results derived from our study, it seems more reasonable that the relationship between $\mathrm{Mg}$ and $\mathrm{OA}$ is mostly dependent on chondrocyte metabolism, rather than inflammation itself. However, this finding should be confirmed by further longitudinal studies.

The study has several limitations: (i) the study sample size is relatively small; (ii) some of the demographic variables associated with knee OA such as body weight and height were unable to be obtained from the medical records; and (iii) the study population was female dominant. The strengths are: (i) all radiographs were assessed by two independent observers and the reliability between assessors was good; and (ii) biochemical analysis of serum $\mathrm{Mg}$ and inflammatory biomarkers was performed nearly at the same time, when the X-rays were undertaken.

In conclusion, serum $\mathrm{Mg}$ level is associated with the radiological severity of knee OA. However, it is not correlated with the inflammatory markers including ESR and CRP in this patient population. Therefore, further, well-designed, clinical studies are needed to identify the exact underlying mechanism of the inverse relation between $\mathrm{Mg}$ and $\mathrm{OA}$. 


\section{Declaration of conflicting interests}

The authors declared no conflicts of interest with respect to the authorship and/or publication of this article.

\section{Funding}

The authors received no financial support for the research and/or authorship of this article.

\section{REFERENCES}

1. Goggs R, Vaughan-Thomas A, Clegg PD, Carter SD, Innes JF, Mobasheri A, et al. Nutraceutical therapies for degenerative joint diseases: a critical review. Crit Rev Food Sci Nutr 2005;45:145-64.

2. Nielsen FH. Magnesium, inflammation, and obesity in chronic disease. Nutr Rev 2010;68:333-40.

3. Barbagallo M, Dominguez LJ, Galioto A, Ferlisi A, Cani C, Malfa L, et al. Role of magnesium in insulin action, diabetes and cardio-metabolic syndrome X. Mol Aspects Med 2003;24:39-52.

4. Touyz RM. Role of magnesium in the pathogenesis of hypertension. Mol Aspects Med 2003;24:107-36.

5. Abbott RD, Ando F, Masaki KH, Tung KH, Rodriguez $\mathrm{BL}$, Petrovitch $\mathrm{H}$, et al. Dietary magnesium intake and the future risk of coronary heart disease (the Honolulu Heart Program). Am J Cardiol 2003;92:665-9.

6. Dai Q, Shrubsole MJ, Ness RM, Schlundt D, Cai Q, Smalley WE, et al. The relation of magnesium and calcium intakes and a genetic polymorphism in the magnesium transporter to colorectal neoplasia risk. Am J Clin Nutr 2007;86:743-51.

7. Leone N, Courbon D, Ducimetiere P, Zureik M. Zinc, copper, and magnesium and risks for all-cause, cancer, and cardiovascular mortality. Epidemiology 2006;17:308-14.

8. Zeng C, Li H, Wei J, Yang T, Deng ZH, Yang Y, et al. Association between Dietary Magnesium Intake and Radiographic Knee Osteoarthritis. PLoS One 2015;10: 127666.

9. Zeng C, Wei J, Li H, Yang T, Zhang FJ, Pan D, et al. Relationship between Serum Magnesium Concentration and Radiographic Knee Osteoarthritis. J Rheumatol 2015;42:1231-6.

10. Qin B, Shi X, Samai PS, Renner JB, Jordan JM, He K. Association of dietary magnesium intake with radiographic knee osteoarthritis: results from a population-based study. Arthritis Care Res (Hoboken) 2012;64:1306-11.

11. Hunter DJ, Hart D, Snieder H, Bettica P, Swaminathan R, Spector TD. Evidence of altered bone turnover, vitamin D and calcium regulation with knee osteoarthritis in female twins. Rheumatology (Oxford) 2003;42:1311-6.

12. Kellgren JH, Lawrence JS. Radiological assessment of osteoarthrosis. Ann Rheum Dis 1957;16:494-502.

13. Baker JF, Byrne DP, Walsh PM, Mulhall KJ. Human chondrocyte viability after treatment with local anesthetic and/or magnesium: results from an in vitro study. Arthroscopy 2011;27:213-7.

14. Feyerabend F, Witte F, Kammal M, Willumeit R. Unphysiologically high magnesium concentrations support chondrocyte proliferation and redifferentiation. Tissue Eng 2006;12:3545-56.

15. Vormann J, Förster C, Zippel U, Lozo E, Günther T, Merker $\mathrm{H}$, et al. Effects of magnesium deficiency on magnesium and calcium content in bone and cartilage in developing rats in correlation to chondrotoxicity. Calcif Tissue Int 1997;61:230-8. 